\title{
COMPUTER GENERATED NATURAL INNER AUTOMORPHISMS OF CAYLEY'S ALGEBRA
}

\author{
by P. J. C. LAMONT
}

(Received 14 March, 1981)

1. Introduction. This paper results from the design and development of computer software for lengthy computations with Cayley's algebra $C$ over the field of reals. The algebra $C$ is 8 -dimensional over the reals and is not associative. Integer elements of $C$ are defined and can be stored as integer arrays. The problem of solving linear equations $\alpha \xi=\beta$ in $C$ is implemented by using the equation $\xi=\bar{\alpha} \beta / N(\alpha)$ where $\bar{\alpha}$ is the conjugate and $N(\alpha)$ is the non-zero norm of $\alpha$. Programming multiplication of Cayley numbers is comparable in difficulty to programming matrix multiplication for matrices with eight rows and eight columns.

In an associative ring with inverses, such as the quaternions over the reals, the mapping

$$
[\sigma]: \xi \rightarrow \sigma \xi \sigma^{-1}
$$

is an automorphism for any invertible $\sigma$ in the ring. The mapping $[\sigma]$ is called inner and the element $\sigma$ is said to induce the automorphism $[\sigma]$. As is shown by the following theorem, which is proved in [3], some but not all of the elements of $C$ of non-zero norm induce automorphisms. Let $R(\sigma)$ denote the real part of $\sigma$.

THEOREM 1.2. An element $\sigma$ of $C$ satisfying

$$
4 R^{2}(\sigma)=N(\sigma) \neq 0
$$

induces an automorphism $[\sigma]$ of $C$.

Products of automorphisms $[\sigma]$, with $\sigma$ satisfying (2), are called natural inner automorphisms of $C$.

2. Development. The images of the standard basis $\left\{i_{s}\right\}_{0}^{7}$ under $[\sigma]$ as in (2) can be obtained for suitable choices of $\sigma$. Records were kept of the images of the basis elements for choices of $\sigma$ as a unit element of an integral subsystem of $C$. For example, $\sigma=\frac{1}{2}\left(1+i_{1}+i_{2}+i_{3}\right)$ gives

$$
\begin{aligned}
& i_{0} \rightarrow i_{0}=1, \\
& i_{1} \rightarrow-i_{3}, \\
& i_{2} \rightarrow i_{1}, \\
& i_{3} \rightarrow-i_{2}, \\
& i_{4} \rightarrow \frac{1}{2}\left(-i_{4}+i_{5}+i_{6}-i_{7}\right), \\
& i_{5} \rightarrow \frac{1}{2}\left(-i_{4}-i_{5}+i_{6}+i_{7}\right), \\
& i_{6} \rightarrow \frac{1}{2}\left(-i_{4}-i_{5}-i_{6}-i_{7}\right), \\
& i_{7} \rightarrow \frac{1}{2}\left(+i_{4}-i_{5}+i_{6}-i_{7}\right) .
\end{aligned}
$$

Glasgow Math. J. 23 (1982) 187-189. 
Further non-trivial automorphisms were obtained for other choices of $\sigma$ from the orders of $C$. For the definition of order see [4].

An automorphism $\theta$ is called a reflection if $\theta^{2}=1$ where $\theta \neq 1$. Moreover, a reflection $\theta$ of $C$ is 1 on some quaternion subalgebra $Q$ of $C$ and -1 on the orthogonal complement of $Q$. The mapping $\theta$ is then called the reflection in $Q$. Every automorphism of $C$ is a product of reflections. This result is detailed in Jacobson [2], a paper in which another definition of inner automorphisms of $C$ is employed. It was natural to enquire whether reflections can be generated as natural inner automorphisms. Linked lists of possible mutiplicands were implemented along with the on-line files to discover the following theorem.

THEOREM 2.1. Let $H$ be a quaternion subalgebra of $C$. Let $H$ have basis $\left\{i_{s}\right\}_{0}^{3}$. Let $\sigma=\frac{1}{2}\left(1+i_{1}+i_{2}+i_{3}\right)$ and $\rho=i_{3} \sigma$. Then $\left[(\sigma \rho)^{-1}\right][\sigma][\rho]$ and $\xi[\sigma]\left[\rho^{2}\right] \zeta^{2}$ both represent the reflection in $H$.

Because of the sparse nature of the arrays produced as images, many techniques described in [1] could be employed. The theorem is proved using the Dickson multiplication

$$
\xi \eta=\xi_{0} \eta_{0}-\bar{\eta}_{1} \xi_{1}+\left(\eta_{1} \xi_{0}+\xi_{1} \bar{\eta}_{0}\right) i_{4}
$$

where $\xi=\xi_{0}+\xi_{1} i_{4}, \eta=\eta_{0}+\eta_{1} i_{4}$, and $\xi_{0}, \xi_{1}, \eta_{0}, \eta_{1} \in H$ and $i_{4} \in H^{\perp}$.

3. Conclusion. We can now prove the following result.

THEOREM 3.1. Every automorphism of $C$ is a natural inner automorphism.

Proof. Let $\tau$ be any automorphism of $C$. Then $\tau=\prod_{r=1}^{n} \theta_{r}$ where $\theta_{r}(1 \leq r \leq n)$ are reflections in quaternion subalgebras $H_{r}$. Choose an orthonormal basis, including 1 , in $H_{r}$. Label this basis $1, i, j, k$ and arrange the basis so that $i(j k)=-1$. Let

$$
\sigma_{r}=\frac{1}{2}(1+i+j+k) \text { and } \rho_{r}=k \sigma_{r} .
$$

The result follows from Theorem 2.1.

Acknowledgement. We wish to thank Monmouth College, Illinois, for the use of their facilities. Programs were written for a PDP-11/40 using MICS/11 operating system under the RSTS/E time sharing monitor in BASIC-PLUS.

\section{REFERENCES}

1. J. R. Bunch and D. J. Rose, Sparse matrix computations (New York, 1976).

2. N. Jacobson, Composition algebras and their automorphisms, Rend. Circ. Mat. Palermo 7 (1958), 55-80. 
3. P. J. C. Lamont, Arithmetics in Cayley's algebra, Proc. Glasgow Math. Assoc. 6 (1963), 99-106.

4. P. J. C. Lamont, Factorization and arithmetic functions for orders in composition algebras, Glasgow Math. J. 14 (1973), 86-95.

QIS DEPARTMENT

WESTERN ILLINOIS UNIVERSTTY

MACOMB, ILLINOIS 61455 